\title{
Distribución potencial de Musca domestica en el municipio de Jesús María, Aguascalientes, con el uso de escenarios de cambio climático
}

Antonio de Jesús Meraz Jiménez ${ }^{\mathrm{a}}$

Armando López Santos ${ }^{b}$

Carlos Alberto García Munguía ${ }^{c}$

Jorge Alejandro Torres González ${ }^{\mathrm{a}}$

Alberto Margarito García Munguía ${ }^{a^{*}}$

${ }^{a}$ Universidad Autónoma de Aguascalientes. Centro de Ciencias Agropecuarias, Jesús María, Aguascalientes, México.

b Universidad Autónoma Chapingo. Unidad Regional Universitaria de Zonas Áridas. Bermejillo, Durango, México.

${ }^{\mathrm{c}}$ Universidad de Guanajuato. Departamento de Veterinaria y Zootecnia, DICIVA. Irapuato, Guanajuato, México.

*Autor de correspondencia: almagamu@hotmail.com

\section{Resumen:}

M. domestica es el mayor parásito doméstico que causa descomposición de alimentos y actúa como vector de más de 100 patógenos para la medicina humana y veterinaria, tiene una cercana asociación con el hombre y su ambiente. El objetivo del presente estudio fue evaluar el desarrollo de $M$. domestica a diferentes niveles de temperatura y humedad relativa y determinar su potencial incidencia en el municipio de Jesús María, Aguascalientes. El estudio se desarrolló en el Centro de Ciencias Agropecuarias de la Universidad Autónoma de Aguascalientes (CCA-UAA), que presenta un clima semiseco templado (BS1k) con 
promedios anuales de temperaturas y precipitación de 16 a $18{ }^{\circ} \mathrm{C}$ y 500 a $600 \mathrm{~mm}$, respectivamente; se evaluaron seis tratamientos con diferentes temperaturas y humedad relativa. Los resultados se analizaron en un diseño completamente al azar y mediante comparación de medias de Tukey $(\alpha=0.005)$. Los resultados muestran que la mosca doméstica se puede desarrollar a temperaturas ambientales de 20 a $30{ }^{\circ} \mathrm{C}$; y con base en el modelo CNRMCM5 (RCP4.5) en diferentes horizontes de tiempo cercano (2015-2039), medio (2040-2060) y lejano (2090) de escenarios de cambio climático, la mosca tendrá una duración de alrededor de tres meses para el horizonte cercano y de cinco meses para el medio y lejano. Con estos datos se provee información importante que permite planear el desarrollo de estrategias para su prevención, monitoreo y control.

Palabras clave: Parásito, Vector, Temperatura, Humedad relativa, Precipitación pluvial.

Recibido: 02/08/2016

Aceptado: 05/03/2018

\section{Introducción}

Musca domestica es un insecto sinantrópico, que tiene una cercana asociación con el hombre y su ambiente; se puede encontrar en cualquier lugar donde existe población humana, y además se asocia con animales de corral, como aves, ganado vacuno, equino y porcino ${ }^{(1)}$, es por ello que tiene un alto potencial en la propagación de diversas enfermedades que pueden pasar de una persona a otra, o bien del animal a la persona ${ }^{(2)}$. Se estima que en los Estados Unidos de América se gastan anualmente 1.6 millones de dólares americanos en insecticidas para el control de este parásito en granjas avícolas ${ }^{(3)}$.

La importancia en la prevención, monitoreo y control de $M$. domestica es debido a que se trata del principal parásito doméstico que causa irritación, descomposición de alimentos y actúa como vector de más de 100 patógenos ${ }^{(4)}$. Además, está implicada en la propagación de alrededor de 30 enfermedades causadas por bacterias y protozoarios, aunque, M. domestica puede prosperar sin causar ninguna infección ${ }^{(5)}$. 
La temperatura es un factor limitante importante para la supervivencia de poblaciones ectotermas, como las moscas, que a menudo permanecen expuestas a las variaciones térmicas extremas en su hábitat natural, sobre todo durante los meses de verano ${ }^{(6)}$. La temperatura de $25{ }^{\circ} \mathrm{C}$ es la más cercana al óptimo desarrollo de la $M$. domestica ${ }^{(7)}$; varios factores del ambiente como la temperatura, precipitación, humedad relativa y el tipo y uso de suelo tienen una influencia directa en la dinámica de sus poblaciones ${ }^{(8)}$.

Está claro que la presencia y persistencia de $M$. domestica puede exacerbarse por el ganado doméstico, como es el caso del estado de Aguascalientes, donde hasta $2015^{(9)}$ registró una gran cantidad de establos que sumaron una población de bovino lechero aproximadamente de 20 mil cabezas. La interacción con áreas urbanas densamente pobladas, por ejemplo para regiones ganaderas como la Comarca Lagunera, semejante al caso de Aguascalientes, y en particular de Jesús María, puede ocasionar problemas de salud pública por la gran cantidad de enfermedades que propaga, por lo que es importante tomar medidas de control de dicho insecto. Por ello, se planteó como objetivo evaluar el desarrollo poblacional de $M$. domestica con variaciones de temperatura y humedad relativa, para determinar su distribución potencial en el escenario de cambio climático CNRMCM5 (RCP4.5) y tres periodos de tiempo futuro, cercano (2015-2039), medio (2045-2069) y lejano (2075-2099) para el municipio de Jesús María del Estado de Aguascalientes.

\section{Material y métodos}

La presente investigación se realizó en el laboratorio de análisis de recursos naturales y sistemas agrarios, del Centro de Ciencias Agropecuarias de la Universidad Autónoma de Aguascalientes (CCA). Se utilizó una incubadora del laboratorio de investigación de clínica veterinaria e invernadero. Las pupas de mosca utilizadas como progenitoras se recolectaron en el área pecuaria del CCA, para su posterior desarrollo en laboratorio.

Las pupas recolectadas, se colocaron en jaulas de $30 \times 30 \times 30 \mathrm{~cm}$ a una temperatura de $24 \pm$ $2^{\circ} \mathrm{C}$, elaboradas con alambre de acero inoxidable y cubiertas con tela tipo tul (100\% nylon) donde se llevó a cabo la reproducción de la mosca; una vez emergidas las moscas, se alimentaron con agua azucarada al $10 \%$ con la que se saturaron pequeñas porciones de algodón, y como sustrato para oviposición y alimentación de las larvas se utilizó una mezcla de agua con salvado de trigo (70 \%), otra de leche en polvo y agua (30\%).

Las pupas obtenidas de las jaulas fueron las que se usaron para el establecimiento del experimento a diferente temperatura y humedad relativa (HR). Cada pupa se colocó en vasos 
de polipropileno de una onza con tapa para evitar la copulación entre hembra y macho. Una vez que emergieron, las moscas fueron sexadas y colocadas en diferentes jaulas (tamaño 30 x 30 x $30 \mathrm{~cm}$ ); de ahí se extrajeron nueve hembras y nueve machos, las cuales se colocaron en tres jaulas (tamaño 10 x 10 x 10) cm de acero inoxidable y cubiertas con tela tul con tres individuos por sexo en cada una. Este diseño fue sometido a seis tratamientos con condiciones diferentes de temperatura y humedad relativa (Cuadro 1).

Cuadro 1: Clave por tratamiento, rangos de temperatura y humedad relativa definidos para el experimento en $M$. domestica

\begin{tabular}{ccc}
\hline Tratamientos & Temperatura $\left({ }^{\circ} \mathbf{C}\right)$ & Humedad relativa $(\%)$ \\
\hline T1 & $27.5 \pm 2.5$ & 90 a 95 \\
T2 & $22.5 \pm 2.5$ & 60 a 65 \\
T3 & $32.5 \pm 2.5$ & 20 a 25 \\
T4 & $32.5 \pm 2.5$ & 35 a 40 \\
T5 & 35 & 90 \\
T6 & $32.5 \pm 2.5$ & 15 a 20 \\
\hline
\end{tabular}

El diseño experimental fue completamente aleatorizado con tres repeticiones. Se evaluaron las variables de número de larvas, pupas y emergencia de mosca por jaula con ayuda del programa STATISTICA ${ }^{\circledR}$ Versión 13.

Además, se utilizó la Red Nacional de Estaciones Estatales Agroclimatológicas, Agroclima $^{(10)}$ del Instituto Nacional de Investigaciones, Forestales, Agrícolas y Pecuarias (INIFAP) para obtener datos de las temperaturas máxima, mínima y media, así como de humedad relativa (HR) para el municipio de Jesús María; con base en ello, se analizó la relación de las variables con el desarrollo poblacional de $M$. domestica y su posible infestación en el área de estudio. Los datos climáticos se interpolaron por el método basado en el inverso de la distancia ponderada (IDW, por sus siglas en inglés), el cual es descrito y aplicado en casos semejantes por diversos autores ${ }^{(11,12)}$.

El primer producto del proceso geoespacial fue la creación de una imagen de barrido (raster) ajustada al máximo y mínimo como valores extremos. El segundo producto fue una imagen clasificada a partir del cambio de sus propiedades en cinco clases asociado a su distribución a nivel de municipal. Las imágenes raster de temperatura mínima se construyeron con base en el modelo creado por el Centro Nacional de Investigación Meteorológica (CNRM, por sus siglas en francés), bajo la nomenclatura: CNRMCM5 (RCP4.5) ${ }^{(13)}$, el cual forma parte del $5^{\circ}$ Informe de Valoración de Cambio Climático del $\mathrm{IPCC}^{(14)}$. Este y otros modelos (GDFLCM3, HADGEM2-ES, etc.), tras ser probados y re-escalados de $0.5^{\circ}$ x $0.5^{\circ}(55 \times 55 \mathrm{~km}$, aproximadamente) a 30” x 30” (926 x 926 m) para su aplicación en la Región México, Sur 
de los Estados Unidos de América y el Caribe, fueron descargados del portal de la Unidad de Informática para las Ciencias Atmosféricas y Ambientales (UNIATMOS) del Centro de Ciencias de la Atmósfera de la Universidad Nacional Autónoma de México (UNAM) ${ }^{(15)}$. El CNRMCM5, se procesó a partir de diferentes horizontes de tiempo futuro: cercano (20152039), medio (2045-2069) y lejano (2075-2099). Para ello, se utilizaron distintos módulos del programa ArcMap 10.2.2 ${ }^{\circledR}$ (ESRI, Redlands, CA).

\section{Resultados y discusión}

\section{Desarrollo de larvas}

Al hacer la comparación entre medias del número de larvas por tratamiento, se observó que el tratamiento uno (T1) fue el mejor con 161 larvas por jaula (Figura 1). Por el contrario, en los tratamientos donde no se presentaron larvas se debió a la variabilidad de la temperatura, ya que el desarrollo de las larvas depende de ésta ${ }^{(16)}$, siendo $8{ }^{\circ} \mathrm{C}$ el umbral para su desarrollo ${ }^{(17)}$. Las larvas prefieren para su desarrollo temperaturas de $35^{\circ} \mathrm{C}$ con alta humedad y cuando están completamente desarrolladas de 15 a $20{ }^{\circ} \mathrm{C}$ con baja HR, y no resisten temperaturas por encima de $\operatorname{los} 45^{\circ} \mathrm{C}^{(18)}$. En otro estudio se reporta que la mayor distribución de larva de mosca en gallinaza ocurre a una temperatura de 17 a $35^{\circ} \mathrm{C}^{(19)}$. 
Figura 1: Comparación de medias del número de larvas de $M$. domestica por tratamiento

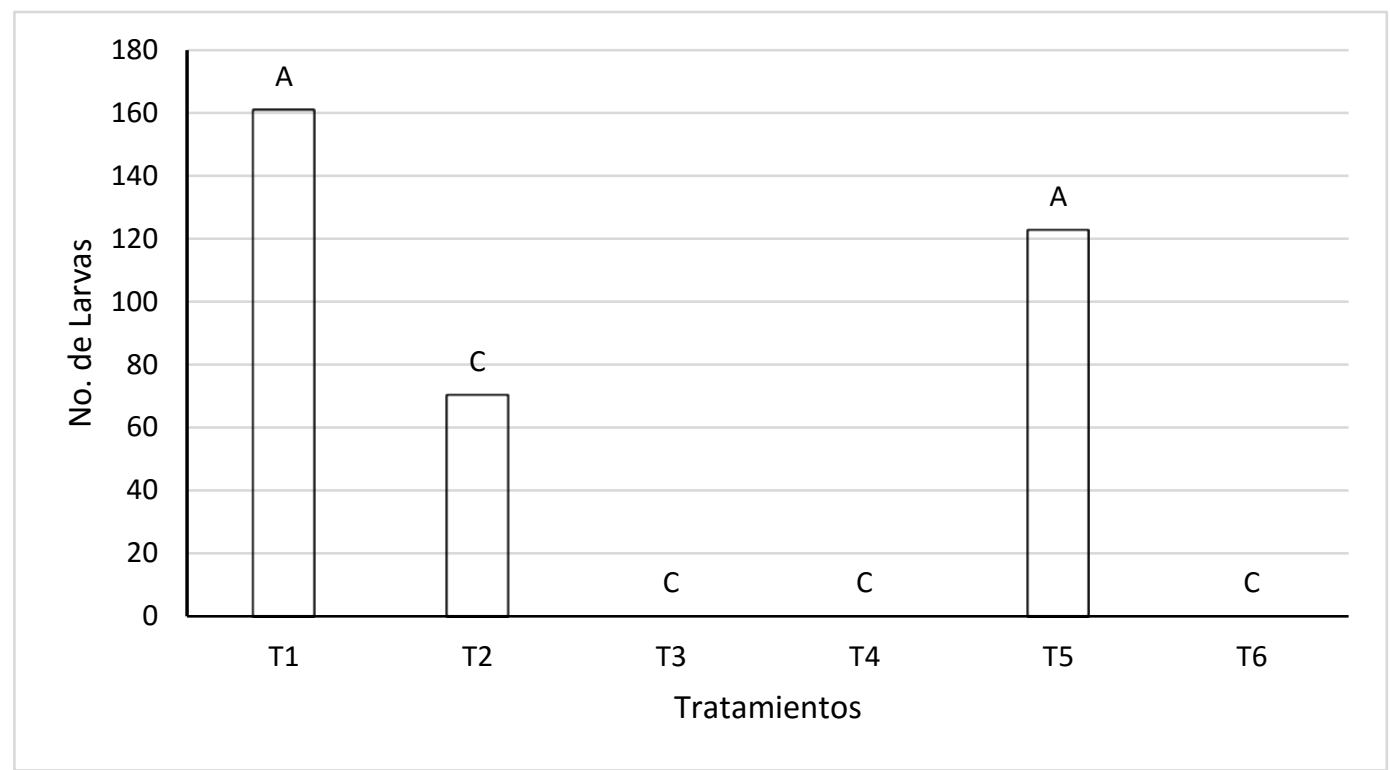

*Valores con la misma letra no presentan diferencia significativa $(P \leq 0.01)$.

El T5 fue el segundo mejor después del T1, con 122 larvas por jaula; la diferencia entre estos dos fue la temperatura más baja para el primero $\left(25\right.$ a $30^{\circ} \mathrm{C}$ para el $\mathrm{T} 1$ y $35^{\circ} \mathrm{C}$ para el T5) y la HR constante para el T5. La humedad es un factor determinante para la sobrevivencia de las larvas, ya que se secan si no hay suficiente, mientras que demasiada humedad conduce a su ahogamiento ${ }^{(20)}$.

\section{Desarrollo larvario a estado de pupas}

En tres de los seis tratamientos (T3, T4 y T6) no hubo presencia de larva debido a que al inicio del experimento las temperaturas no permitieron el desarrollo del huevecillo. Debido a que esos tratamientos están por encima de los $30^{\circ} \mathrm{C}$ con baja humedad relativa, y la máxima ovoposición $M$. domestica está entre los 25 a $30{ }^{\circ} \mathrm{C}$ y los huevecillos deben de permanecer húmedos o no eclosionarán ${ }^{(21)}$.

En el paso del estadio larvario a pupa, nuevamente el T1 fue mejor que los demás tratamientos, ya que sobrevivieron 115 pupas en promedio por jaula de las 160 larvas, seguido del T2 con 62 pupas de 72 larvas por jaula (Figura 2), en el T5 solo el $9.5 \%$ 
sobrevivió de larva a pupa (12 pupas de 122 larvas); esto se pudo deber a la alta HR que fue del $90 \%$ a las que fueron expuestas; mismo efecto fue observado en un estudio en donde la máxima mortalidad se presentó al llegar al $100 \%$ de HR y una temperatura superior a los $30{ }^{\circ} \mathrm{C}^{(22)}$. La pupa tolera menor humedad que la larva y puede desarrollarse a temperatura de 35 a $40{ }^{\circ} \mathrm{C}$ pero solo en un periodo mínimo de 3 a 4 días ${ }^{(18)}$, mientras que en este estudio, las pupas permanecieron por 16 días y a temperaturas $35^{\circ} \mathrm{C}$. En un estudio se encontró que a temperaturas de $48^{\circ} \mathrm{C}$ (por $15 \mathrm{~min}$ ) se eliminan todas las etapas del ciclo de vida de la mosca, a $37{ }^{\circ} \mathrm{C}$ sobrevivieron por poco más de $4 \mathrm{~h}$ y a $42{ }^{\circ} \mathrm{C}$ y solo afectó a los adultos (aunque solo fueron expuestos durante una hora aproximadamente), mientras que en el caso de las pupas se observó resistencia hasta 44 a $46{ }^{\circ} \mathrm{C}^{(23)}$. En dicho estudio los periodos a los que fueron sometidos los tratamientos fueron relativamente cortos (de algunas horas), mientras que en este estudio se sometieron a las diferentes condiciones de temperatura y HR hasta completar el ciclo de vida de la mosca, que fue de 19 a 22 días. Se realizó un análisis de varianza entre tratamientos para comparar el porcentaje de larva transformada a pupa y se encontró que los tratamientos uno y dos fueron los mejores con el 74 y $86 \%$ de pupas respectivamente.

Figura 2: Comparación de medias del número de pupas de $M$. domestica por tratamiento.

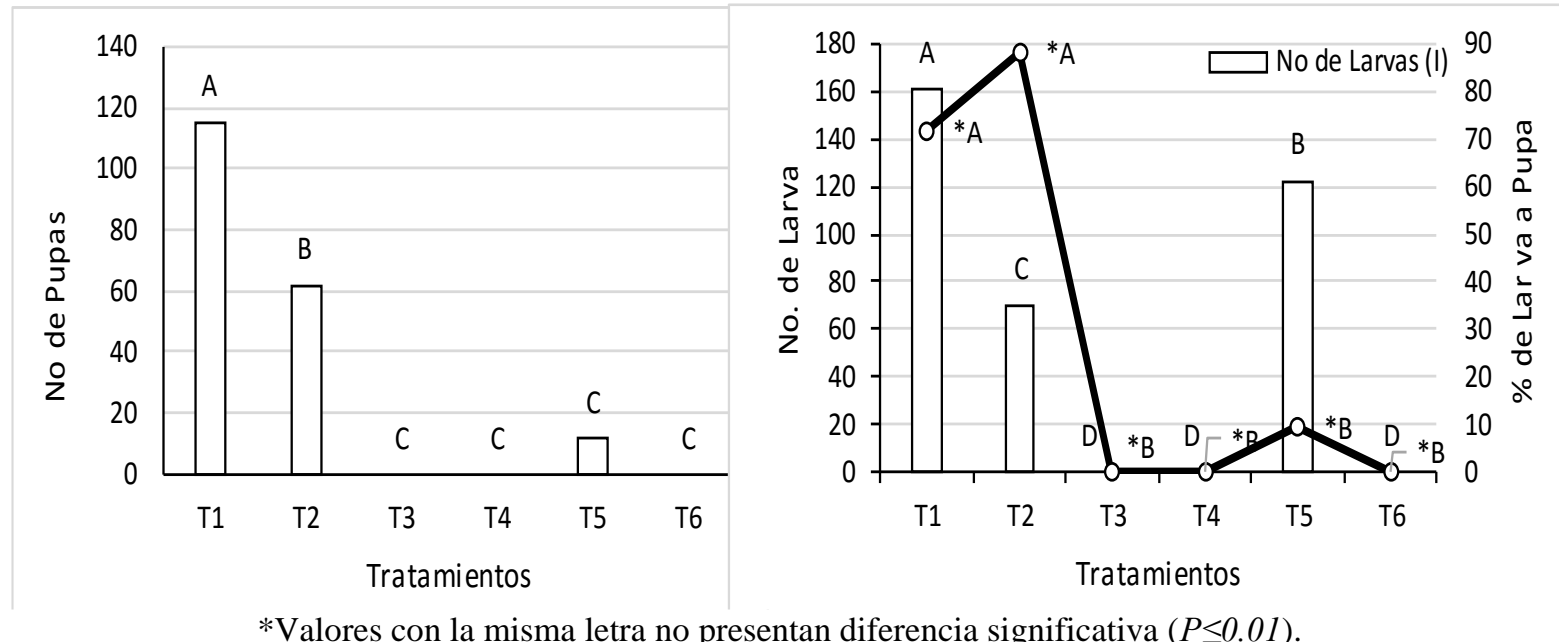

\section{Cambio de estado pupa a estado adulto}

En relación a la emergencia de los adultos, el tratamiento 1 fue el mejor, por tener la mayor emergencia de adultos por jaula, con 85, le siguió el tratamiento 2 con 54 adultos (Figura 3 ). 
De lo anterior se deduce que la temperatura ideal para el desarrollo de las mosca en este estudio fue de 20 a $30{ }^{\circ} \mathrm{C}$. En otro estudio se observó una alta densidad de moscas a una temperatura media de 20 a $25^{\circ} \mathrm{C}$ y no se detectó presencia de mosca en temperaturas mayores de $45^{\circ} \mathrm{C}$ ni en menores de $10{ }^{\circ} \mathrm{C}^{(17)}$. De igual manera, en un estudio similar se encontró que el mayor número de moscas se dio a temperaturas entre los 25 y $35^{\circ} \mathrm{C}^{(24)}$, por lo que el rango observado en este estudio está dentro de lo reportado por dichos estudios.

Figura 3: Comparación de medias del número de adultos de $M$. domestica por tratamiento
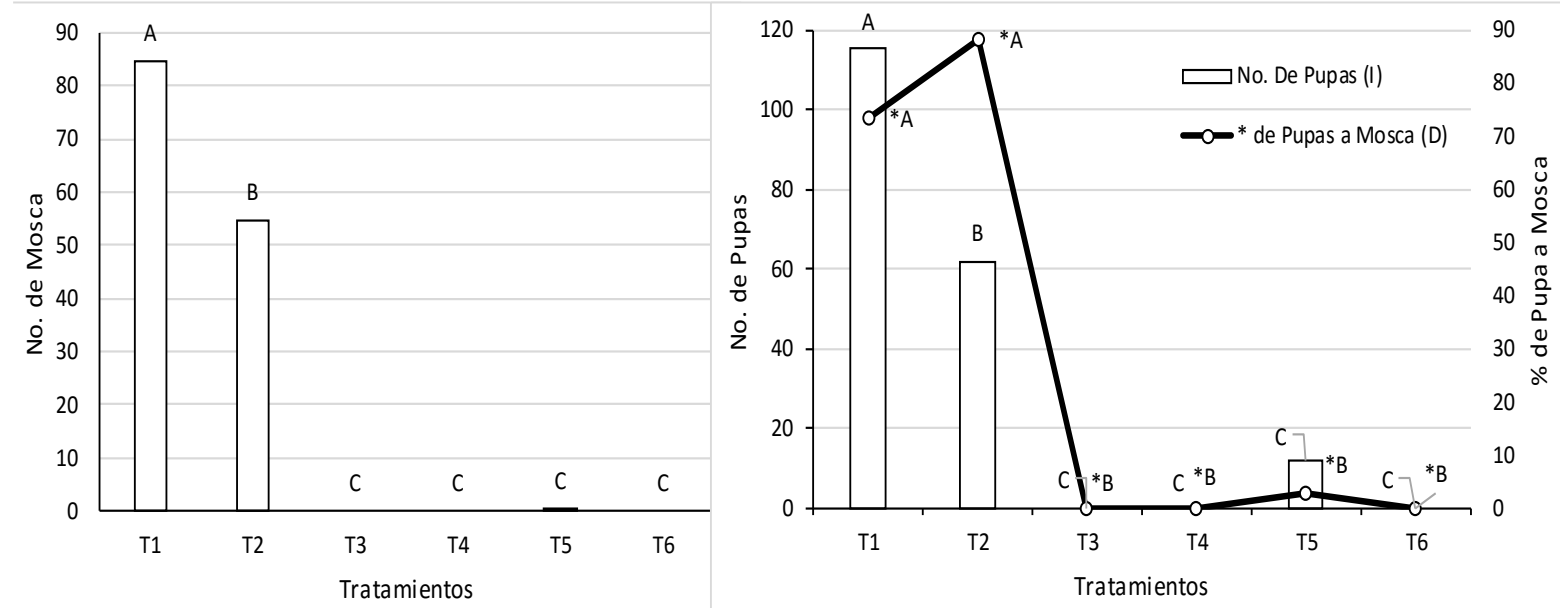

*Valores con la misma letra no presentan diferencia significativa $(P \leq 0.01)$.

Al realizar el análisis de la transformación de pupa a adulto, el tratamiento 2 y 1 porcentualmente son mejores con el 84 y $74 \%$ respectivamente de emergencia (54 y 85 adultos respectivamente). En el caso del T2 con una HR de $62.5 \pm 2.5 \%$ se acerca a lo reportado en estudios similares, donde mencionan que la HR óptima para el desarrollo de la mosca es de 65 a $75 \%{ }^{(22)}$. Es por eso que este tratamiento es más consistente en cuanto al desarrollo de sus diferentes etapas. En el caso del tratamiento cinco, tanto el número de pupas (11) como el de adultos (1) fueron mínimos (Figura 3).

\section{Cambio de estado larvario a estado adulto}

En el análisis del porcentaje del desarrollo larvario al estado adulto, el tratamiento 2 y 1 estadísticamente fueron los mejores con el 73 y $55 \%$ de adultos respectivamente (Figura 4). 
Figura 4: Comparación del número de larvas de $M$. domestica y el porcentaje de larva a adulto

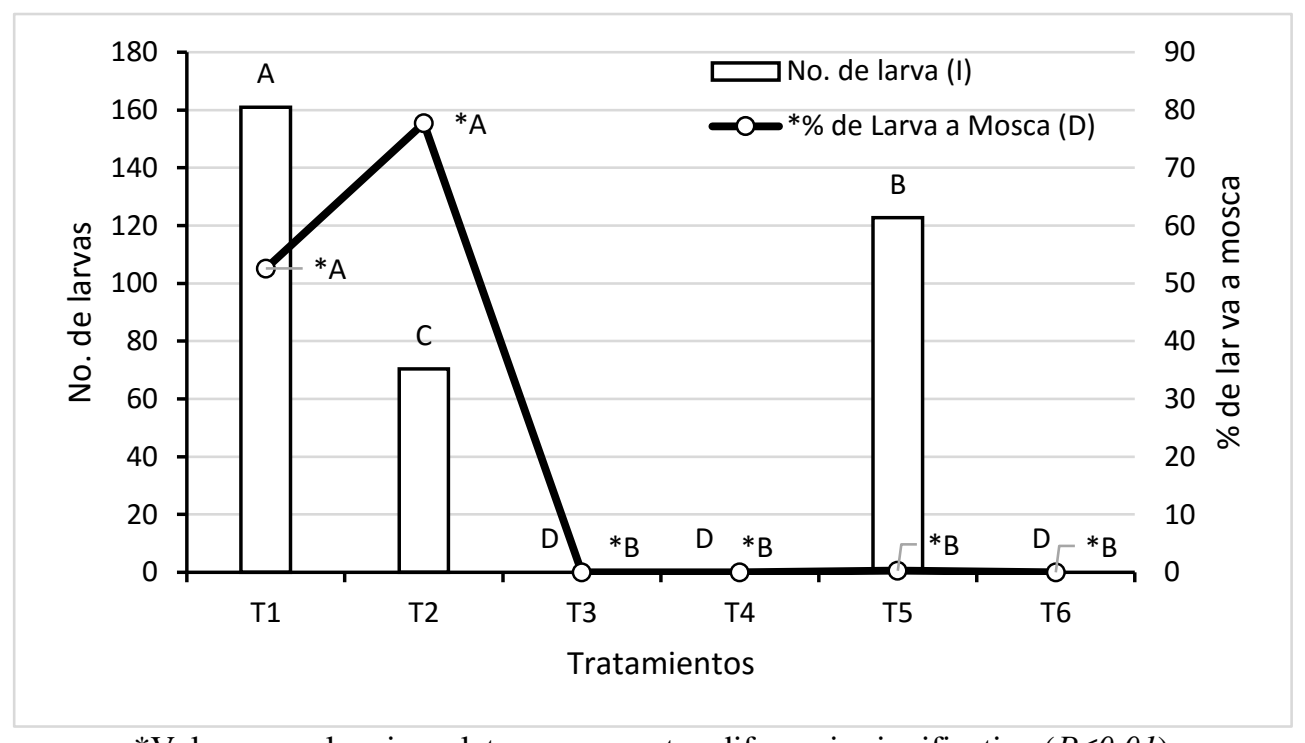

*Valores con la misma letra no presentan diferencia significativa $(P \leq 0.01)$.

\section{Relación entre la temperatura y humedad y las condiciones de desarrollo de M. domestica}

Al relacionar los datos de la temperatura y la humedad relativa en el área del CCA durante el año 2015, con la presencia de $M$. domestica, y los resultados obtenidos en este estudio, se estima que el aumento de la población de la mosca se dio en los meses de junio (con una temperatura mínima extrema de $11.8^{\circ} \mathrm{C}$ ) a agosto (temperatura mínima extrema de $10{ }^{\circ} \mathrm{C}$ ), como se muestra en la Figura 5; siendo esta última temperatura el límite para la eclosión de los huevos y en la que la mosca adulta prácticamente no puede volar ${ }^{(17)}$, por ende se infiere que hay poca copulación. Como regla general la ovoposición no tiene lugar por debajo de $15{ }^{\circ} \mathrm{C}^{(18)}$, lo cual hace que en los meses de abril y mayo las poblaciones de mosca se mantienen bajas en comparación con los meses con mayor temperatura. Con estos datos se podrá determinar el comportamiento de la mosca, ya que como se ha dicho, ésta entra en mayor activad a temperaturas altas $\left(30 \mathrm{a} 33^{\circ} \mathrm{C}\right)$ y humedad relativa baja; por el contrario disminuye su actividad a temperaturas y humedad altas ${ }^{(17,25)}$. En otro trabajo se observó que la abundancia de la mosca se vio influida únicamente por los cambios de lluvia y humedad relativa, ya que la temperatura se mantuvo prácticamente constante durante el año ${ }^{(24)}$. De la 
misma manera, en explotaciones equinas el tamaño de la población de mosca se vio afectada por la temperatura y la humedad, ya que la población de moscas aumenta en primavera cuando las temperaturas suben y disminuye en otoño cuando las temperaturas bajan ${ }^{(26)}$. Se ha observado que la temperatura tiene un gran impacto en la actividad física de $M$. domestica durante el día, la actividad se incrementa con la temperatura a intervalo de 10 a $30-35{ }^{\circ} \mathrm{C}$, además de que se incrementa la dispersión y transmisión de patógenos, y la actividad física disminuye cuando la temperatura está por arriba de $35^{\circ} \mathrm{C}^{(27)}$. Esto indica que la mosca tiene un amplio rango de temperaturas para realizar su actividad; esta información se puede utilizar para establecer medidas preventivas y evitar algún brote de enfermedades.

Figura 5: Temperaturas y humedad relativa para el municipio de Jesús María durante el año 2015

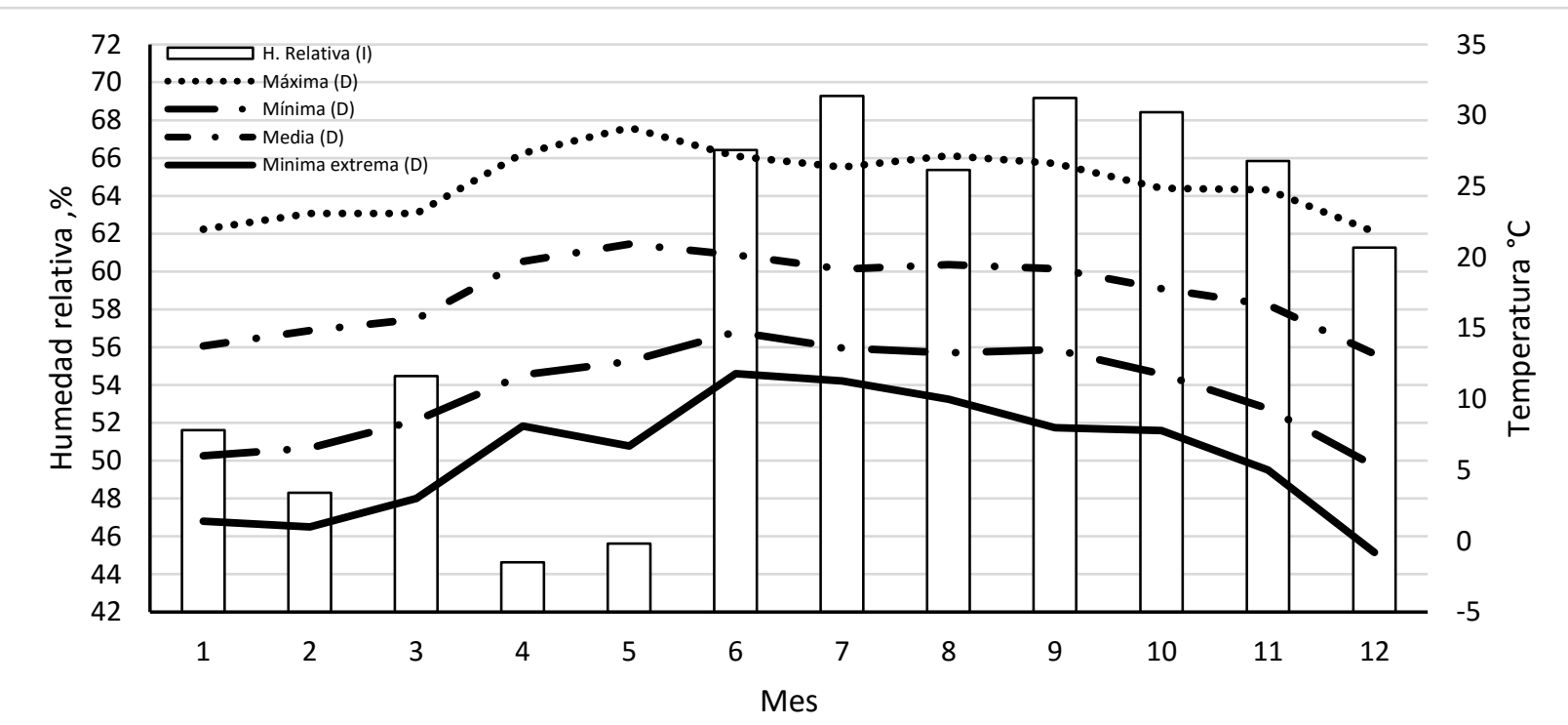

Fuente: Red Nacional de Estaciones Estatales Agroclimatológicas, Agroclima (INIFAP).

\section{Modelo de proyección de temperaturas mínimas}

El conocer las temperaturas mínimas extremas que se presentarán en el futuro en el municipio de Jesús María, permitirá predecir en qué momento la mosca tiene las condiciones ideales para su desarrollo, para esto, se tomó como base el modelo CNRMCM5. RCP45, desarrollado por la UNAM. Los resultados de este experimento muestran que la temperatura es el valor más significativo que induce la mortalidad ${ }^{(22)}$ de $M$. domestica, mientras que la humedad 
relativa jugó un papel importante en la regulación de la viabilidad, pero a un rango moderado de temperatura.

Para el caso del horizonte cercano, se mantendrán las condiciones actuales, es decir, con el desarrollo de la mosca durante los meses de junio a agosto, mientras que para los horizontes mediano y lejano, se incrementarán dos meses las condiciones favorables para el desarrollo de la mosca, de mayo a septiembre (Figura 6). Las proyecciones anteriores van a depender igualmente de otros factores como el viento, alimento, luz y la humedad relativa de la zona. Con este resultado se puede prever que las poblaciones de $M$. domestica, se mantendrán estables hasta el año 2039. En el caso del Reino Unido utilizaron modelos de escenarios de cambio climático y predicen que la población de la mosca podría tener aumentos de hasta el $244 \%$ en 2080 con incrementos en los meses de verano ${ }^{(28)}$.

Figura 6: Proyección de las temperaturas mínimas extremas en diferentes horizontes cercano 2015-2039, medio 2045-2069, lejano 2075-2099 e INIFAP 2015, del municipio de Jesús María

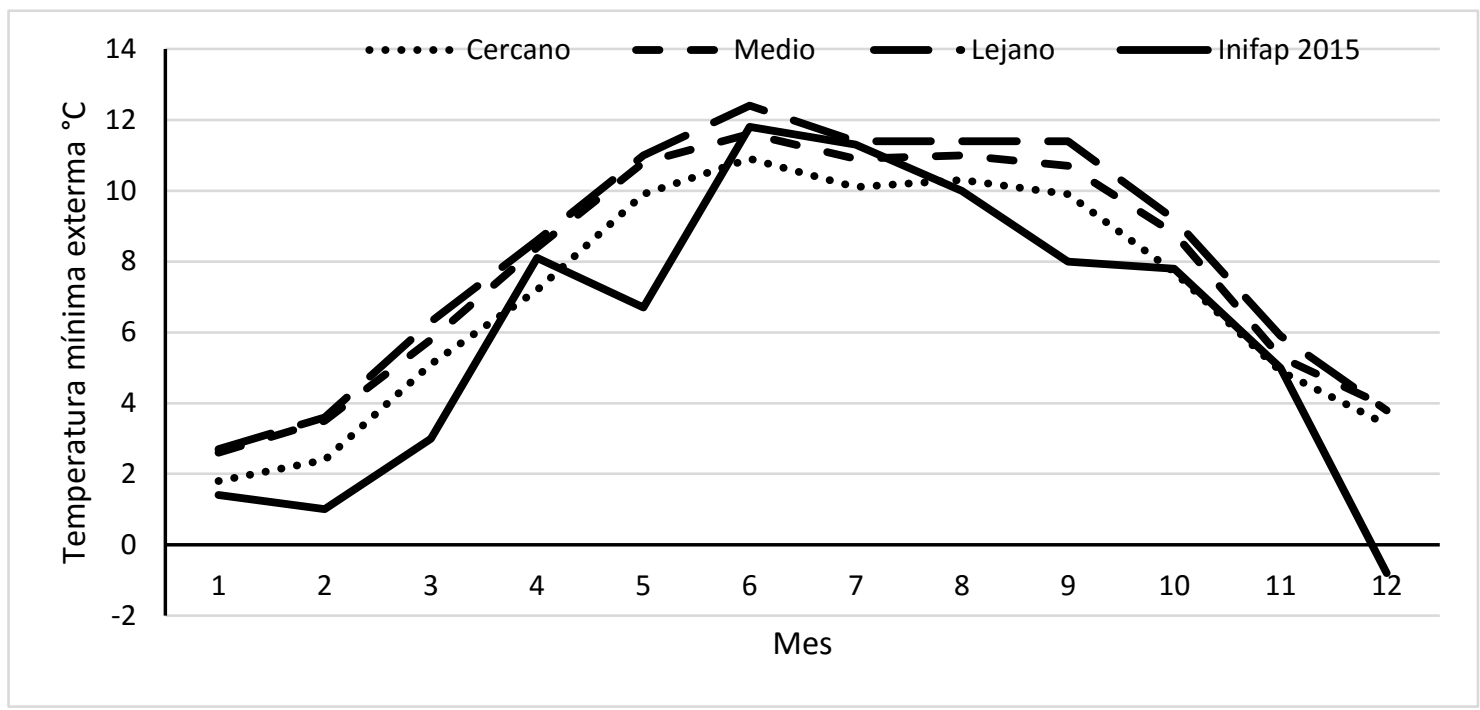

Fuente: $\left({ }^{15}\right)$ y Red nacional de estaciones estatales agroclimatológicas, Agroclima INIFAP.

Con esta información se puede contribuir para establecer programas de control en periodos más favorables para el desarrollo de las moscas y evitar afectaciones en explotaciones pecuarias; en la agroindustria, disminuir la contaminación de alimentos y la propagación de enfermedades a la población. La mosca tiene un alto potencial reproductivo, por lo que requiere de buenas prácticas de control para proteger la salud humana y animal ${ }^{(29)}$. Los métodos de control de la mosca se investigan en países desarrollados, sobre todo los que van dirigidos a granjas e industrias lácteas, donde se formulan los agentes de control y estos son igualmente aplicados en asentamientos humanos y zonas rurales ${ }^{(1)}$. En los casos en que se 
vayan a realizar aplicaciones de algún insecticida para el control de la mosca, estos datos ayudarán a programar dichas actividades y hacerlas más eficaces. En esta proyección no se toman en cuenta los factores bióticos como predadores y parasitoides que se pueden llegar a presentar en próximos años ${ }^{(28)}$.

En la (Figura 7) se muestra la distribución potencial de los diferentes rangos de temperatura en el municipio de Jesús María, donde se observa que cerca de 100,000 habitantes de las 235 localidades del municipio (INEGI $2010^{(30)}$ ), se verán afectadas por el desarrollo poblacional de mosca. El problema se incrementará en las zonas de tonos rojos en donde se concentra la mayor parte de la población (219 localidades con un total de 99,046 habitantes). La M. domestica es a menudo asociada con el ganado de granjas e instalaciones de eliminación de residuos domésticos, donde la acumulación de materia orgánica proporciona condiciones de cría apropiadas $^{(28)}$. Una de las características de la mosca constituye sus preferencias de alimentación, ya que son atraídas por la descomposición de materia vegetal y animal; esto la pone en contacto con los organismos patógenos presentes en los desechos de basura y animales, de donde su potencial riesgo como vector de enfermedades a los seres humanos ${ }^{(31)}$.

Figura 7: Distribución de la temperatura mínima promedio anual de 2015-2039 en el municipio de Jesús María

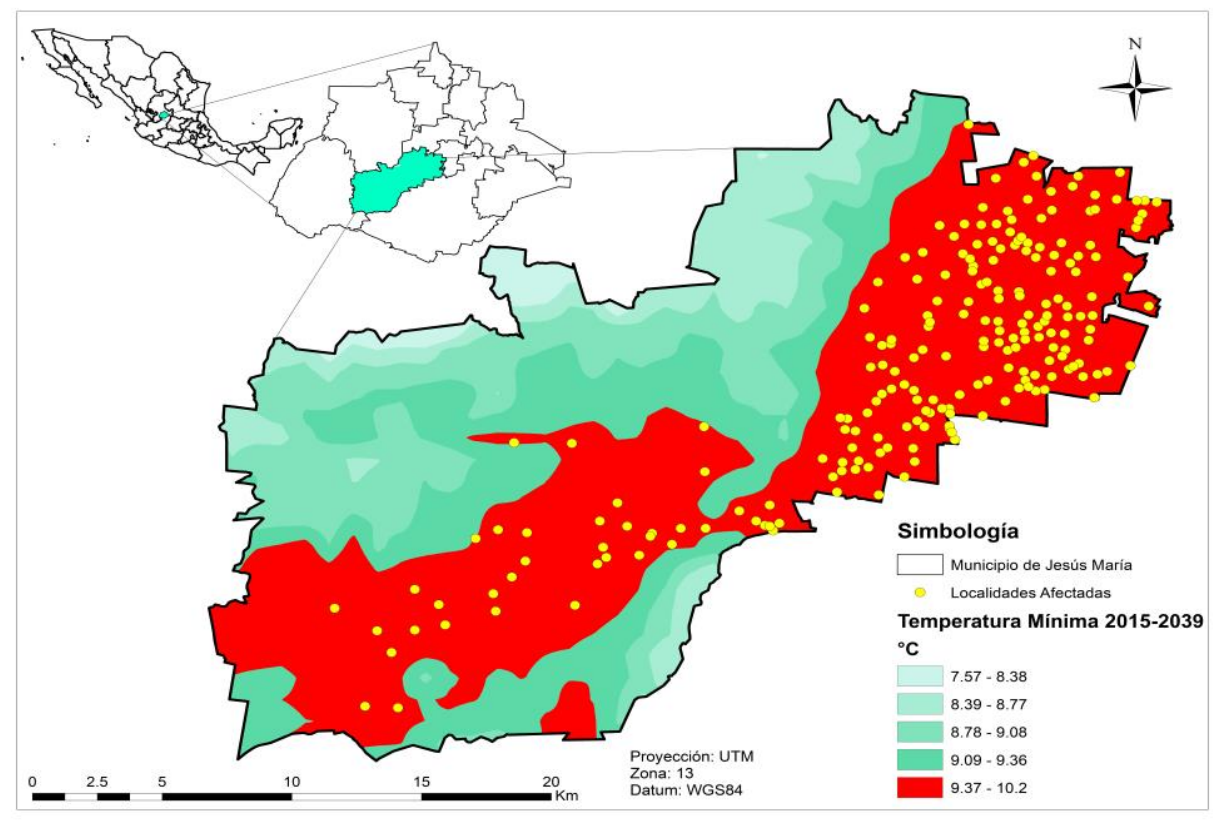

El desarrollo de $M$. domestica en sus diferentes etapas fue afectado por la temperatura y humedad relativa a las que fue expuesta, ya que en el análisis de varianza se encontraron efectos altamente significativos entre los tratamientos para las tres etapas (larva, pupa y 
adulto). Cabe destacar que en algunos tratamientos no se tuvo presencia de larvas, por lo que su valor fue cero, ya que presentaron temperaturas por encima de $42{ }^{\circ} \mathrm{C}$ provocando la muerte de la mosca y que los huevecillos no se desarrollaran.

\section{Conclusiones e implicaciones}

La $M$. domestica se pudo desarrollar a temperaturas de 20 a $30{ }^{\circ} \mathrm{C}$, durante un periodo actual de tres meses al año (de junio a agosto), y con los análisis de los datos climáticos para el caso del municipio de Jesús María, se prevé que la presencia de moscas se verá favorecida por las proyecciones de temperaturas del horizonte medio y lejano, ya que el rango en el cual se puede presentar se extenderá del mes de mayo a septiembre. Esta afectación potencial incluye a prácticamente la totalidad de las áreas pobladas del municipio, por lo que constituye un riesgo que debe considerarse para diseñar medidas de monitoreo y control, por los problemas de contaminación en explotaciones pecuarias, en la agroindustria y en alimentos, que favorece la propagación de enfermedades en la población.

\section{Literatura citada:}

1. Malik A, Singh N, Satya S. House fly (Musca domestica): A review of control strategies for a challenging pest. J Env Sci \& Health, Part B 2007;42(4):453-469. doi: http://dx.doi.org/10.1080/03601230701316481.

2. Mehrabi MR, Zoghimofrad I, Mazinani M, Akbarzadeh, A, Rahimi A. A study of the effect of Bacillus thuringiensis serotype H14 (subspecies israelensis) delta endotoxin on Musca larva. Turk J Med Sci 2015;45(4):794-799. doi: http://dx.doi.org/10.3906/sag1406-91.

3. Burgess ER, King BH. Compatibility of the parasitoid Wasp Spalangia endius (Hymenoptera: Pteromalidae) and insecticides against Musca domestica (Diptera: Muscidae) as evaluated by a new index. J Econ Entomol 2015;108(3):986-992. doi: http://dx.doi.org/10.1093/jee/tov104. 
4. Kumar P, Mishra S, Malik A, Satya S. Insecticidal evaluation of essential oils of Citrus sinensis L. (Myrtales: Myrtaceae) against housefly Musca domestica L. (Diptera: Muscidae). Parasitol Res 2012;(110):1929-1936.

5. Lu X, Shen J, Jin X, Ma Y, Huang Y, Mei H, Zhu J. Bactericidal activity of Musca domestica cecropin (Mdc) on multidrug-resistant clinical isolate of Escherichia coli. Appli Microbiol \& Biotechnol 2012;95(4):939-945. doi: http://dx.doi.org/10.1007/ s00253-011-3793-2.

6. Sharma S, Rohilla MS, Tiwari PK. Developmental and hyperthermia-induced expression of the heat shock proteins HSP60 and HSP70 in tissues of the housefly Musca domestica: An in vitro study. Genet Mol Bio 2007;30(1):159-168.

7. Chapman JW, Goulson D. Environmental versus genetic influences on fluctuating asymmetry in the housefly, Musca domestica. Biol J Linn Soc 2000;(70):403-413. doi: http://dx.doi.org/10.1006/bij1.1999.0408.

8. Kassem HA, El-Sayed YA, Baz MM, Kenawy MA, El Sawaf BM. Climatic factors influencing the abundance of Phlebotomus papatasi (Scopoli) (Diptera: Psychodidae) in the Nile Delta. J Egypt Soc Parasitol 2009;39(1):305-316.

9. SIAP. Servicio de Información Agroalimentaria y Pesquera. 2015. Resumen municipal pecuario. http://www.siap.gob.mx/ganaderia-resumen-municipal-pecuario/. Consultado 1 Jul, 2016.

10. INIFAP. Instituto Nacional de Investigaciones Forestales Agrícolas y Pecuarias. Red Nacional de Estaciones Estatales Agroclimatológicas, Agroclima del Inifap. http://clima.inifap.gob.mx/redinifap/\#. Consultado 6 Ene, 2016.

11. López-Santos A, Pinto-Espinoza J, Ramírez-López EM, Martínez-Prado MA. Modeling the potential impact of climate change in northern Mexico using two environmental indicators. Atm 2013;(26):479-498. http://www.journals.unam.mx/index. php/atm/article/view/32016/38321. Consultado 18 Ago, 2015.

12. López SA, Pinto JE, Esquivel GA, Randeles VHR, Bueno PH. Escenarios climáticos locales basados en los MGCG del IPCC. 1ra ed. Durango, México: Universidad Autónoma Chapingo; 2015. ISBN: 978-607-12-0403-5.

13. Voldoire A, Sanchez-Gomez E, Salas y Mélia D, Decharme B, Cassou C, Sénési S. et al. The CNRM-CM5.1 global climate model: description and basic evaluation. Clim Dyn 2013;(40):2091-2121. doi: http://dx.doi.org/10.1007/s00382-011-1259-y.

14. Edenhofer O, Pichs-Madruga R, Sokona Y, Farahani E, Kadner S, Seyboth K. et al. Contribution of Working Group III to the Fifth Assessment Report of the 
Intergovernmental Panel on Climate Change. In: IPCC. Intergovernmental Panel on Climate Change editor. Climate Change 2014 Mitigation of Climate Change. 1rst ed. United Kingdom and New York, NY, USA: Cambridge University Press; 2014.

15. Fernandez-Eguiarte A, Zavala-Hidalgo J, Romero-Centeno R, Trejo-Vázquez RI. Actualización de los escenarios de cambio climático para estudios de impactos, vulnerabilidad y adaptación. Centro de Ciencias de la Atmósfera. Universidad Nacional Autónoma de México. Instituto Nacional de Ecología y Cambio Climático. Secretaria de Medio Ambiente y Recursos Naturales de México. 2014. http://atlasclimatico. unam.mx:8550/geonetwork/srv/spa/main.home. Consultado 17 Mar, 2016.

16. Sukontason K, Piangjai S, Siriwattanarungsee S, Kabkaew L, Sukontason. Morphology and developmental rate of blowflies Chrysomya megacephala and Chrysomya rufifacies in Thailand: application in forensic entomology. Parasitol Res 2008;102(6):1207:1216. doi: http://dx.doi.org/10.1007/s00436-008-0895-6.

17. Stafford KC. A Guide to biology, dispersal, and management of the housefly and related flies for farmers, Municipalities, and Public Health Officials. 2008 Bulletin 1013. Conn Agr Exp Sta, New Haven http://www.ct.gov/caes/lib/caes/documents/publications/ bulletins/b1013.pdf. Consultado 5 Nov, 2015.

18. WHO. World Health Organization. The housefly: Training and information guide. Vector control series Geneva; 1991. http://www.who.int/iris/handle/10665/58637. Consultado 9 Oct, 2015.

19. Stanfford KC, Bay DE. Dispersion pattern and association of housefly, Musca domestica (Diptera: Muscidae), larvae and both sexes of Macrocheles muscaedomesticae (Acari: Macrochelidae) in response to poultry manure moisture, temperature, and accumulation. Environ Entomol 1987;16(1):159-164. doi: http://dx.doi.org/10.1093/ee/16.1.159.

20. Feldmeyer B, Kozielska M, Kuijper B, Weissing FJ, Beukeboom LW, Pen I. Climatic variation and the geographical distribution of sex-determining mechanisms in the housefly. Evol Ecol Res 2008;10:797-809.

21. Capinera JL. House fly, Musca domestica L. (Diptera: Muscidae). In: Capirera JL editor. Encyclopedia of entomology. 2nd ed. Springer; 2008:1877-1880. doi: 10.1007/978-14020-6359-6.

22. Mishra S, Kumar P, Malik A. Effect of temperature and humidity on pathogenicity of native Beauveria bassiana isolate against Musca domestica L. J Parasit Dis 2015;39(4):697-704. doi: http://dx.doi.org/10.1007/s12639-013-0408-0.

23. Tiwari PK, Archana J, Mohan DRK. Thermotolerance and heat shock response in Musca domestica. Curr Sci 1997;72(7):501-506. 
24. Bong LJ, Zairi J. Temporal changes in the abundance of Musca domestica Linn (Diptera: Muscidae) in poultry farms in Penang, Malaysia. Trop Biomed 2009;26(2):140-148.

25. Dakshinamurty S. The common House-fly, Musca domestica, L., and its behaviour to temperature and humidity. Bull Entomol Res 1948;(39):339-357. doi: https://doi.org/10.1017/S000748530002246X.

26. Machtinger TE, Geden JCh, Kaufman EP, House MA. Use of pupal parasitoids as biological control agents of filth flies on equine facilities. J Integr Pest Manage 2015;6(1):1-16. doi: http://dx.doi.org/10.1093/jipm/pmv015.

27. Schou TM, Faurby S, Kjærsgaard A, Pertoldi C, Loeschcke V, Hald B, Bahrndorff S. Temperature and population density effects on locomotor activity of Musca domestica (Diptera: Muscidae). Environ Entomol 2013;42(6):1322-1328. doi: http://dx.doi.org/10.1603/EN13039.

28. Goulson D, Derwent 1C, Hanley ME, Dunn DW, Abolins SR. Predicting calyptrate fly populations from the weather, and probable consequences of climate change. J Appl Ecol 2005;42(5):795-804. doi: http://dx.doi.org/10.1111/j.1365-2664.2005.01078.x.

29. Selem GS, El-Sheikh EA. Toxicity and biochemical effects of Neem Azal T/S, willow (Salix aegyptiaca L.) and Chasteberry (Vitex agnus-castus L.) on housefly, Musca domestica L. (Diptra: Muscidae). J Biopest 2015;8(1):37-44. ISSN: 0974-391X.

30. INEGI. Instituto Nacional de Estadística y Geografía. Vectorial de localidades amanzanadas y números exteriores, rurales, cierre de planeación del censo de población y vivienda. Jesús María, Aguascalientes. México. 2010.

31. Butler JF, Garcia-Maruniak A, Meek F, Maruniak JE. Wild Florida house flies (Musca domestica) as carriers of pathogenic bacteria. Fla Entomol 2010;93(2):218-223. doi: http://dx.doi.org/10.1653/024.093.0211. 